\title{
Preservation of Arachis Hypogea L. Food Seeds by Cuminumcyminum L. Essential Oil
}

\section{Narendra Kumar*}

Amity Institute of Biotechnology, Amity University Haryana, Gurgaon Manaser-122413, Haryana, India

\begin{abstract}
Samples of groundnut seeds were collected from stores and examined for their associated mycoflora and insects. Fifteen species of fungi were identified by blotter method and 12 species of fungi by agar plate method. In vitro volatile constituents extracted in the form of essential oils from 32 plant species were evaluated against the dominant fungi, Aspergillus flavus and Aspergillus niger. The 2 commercial fungicides was assessed for their antifungal activity against allisolated fungi.

The oil of Cuminumcyminum (Apiaceae) exhibited the greatest toxicity. The oil was found to be fungicidal and thermostable at its minimum inhibitory concentration (MIC) of $400 \mathrm{ppm}$. The oil was characterized by the determination of its various physico-chemical properties. In vivo studies depict that the oil as seed dressing agent and as a fumigant was able to preserve the groundnut food seeds completely for 6 months at 0.50 and $0.76 \mathrm{~mL}$ in containers of $500 \mathrm{~mL}$ capacity holding $400 \mathrm{~g}$ seeds with minimal changes in organoleptic behavior of food seeds during storage. It did not exhibit any adverse effect on seed germination, seedling growth and general health and morphology of plants. GC and GC-MS analysis of the oil revealed recognition of p-mentha-1, 4-dien-7-al (27.4\%), $\mathrm{Y}$-terpinene $(12.8 \%)$, $\beta$-pinene $(11.4 \%)$ and cuminaldehyde $(16.1 \%)$ as major compounds.
\end{abstract}

Keywords: Arachis hypogea L; Cuminumcyminum seed oil; Storage deterioration

\section{Introduction}

Arachis hypogea (peanut, groundnut), an annual oil seed belonging to the Leguminosae family and the Papillionaceae subfamily, is a legume native to South America but now grown in diverse environments in six continents between latitudes 40 degrees $\mathrm{N}$ and 40 degrees S. Arachis hypogea can grow in a wide range of climatic conditions [1]. In a seed production programme, storage of seeds until the distribution during next season assumes paramount importance [2].

A large number of fungi have been reported on seeds of Arachis hypogea [3].The current study concerned storage of groundnut seeds in rural areas where poor storage practice leads to heavy deterioration caused by fungi and insects. Joel-Coats [4] highlighted that Synthetic pesticides brought a new order of insect control, but also a new college of risks. At present, only two fumigants are in common use, methyl bromide and phosphine. Methyl bromide has been identified as a major contributor to ozone depletion, which casts a doubt on its future use in pest control. There have been repeated indications that certain pests have developed resistance to phosphine and metylbromide, so its use is in much suspense. New questions have arisen regarding environmental quality, especially contamination of water air and soil by a host of chemicals some of which are pesticides or their degradation products. In view of the problems with the current fumigants, there is a global interest in alternative strategies including development of chemical substitutes. The interest has been shown inplant products, i.e., essential oils for fumigant action.

The in vivo efficacy of the Cuminumcyminum $\mathrm{L}$ seed oil as a seeddressing agent and fumigant of higher plant origin in the preservation of food seeds of Arachis hypogea was compared synthetic fungicides and fumigants and its physic chemical properties and GC MS analysis were done in order to know major compounds.

\section{Materials and Methods}

\section{Stored seed collection}

Food samples of Arachis hypogea that had been in storage for between 6-8 months were collected. Twenty-five farmer places were visited for collection of stored food seeds.

\section{Mycobiota of stored food seeds of Arachis hypogea}

The mycobiota of stored food seeds of groundnut was studied through agar plate [5] using czapekdox agar medium and standard blotter [6] techniques. Fungal identifications were confirmed following keys and description given by Raper and Thom [7], Gilman [8], Raper and Fennell [9], Booth [10] and Ellis [11,12].

\section{Effect of storage fungi on Arachis hypogea food seeds}

The fungi isolated from food seeds were tested in terms of seed germination and mortality. The fungal species were cultured in czapeks solutions for 15 days at $28 \pm 2^{\circ} \mathrm{C}$ in stationary conditions. The cultures were filtered through whattman no-1 filter paper. Freshly harvested surface sterilized $(0.1 \%$ sodium hypochlorite solution) and washed (sterilized water) seeds were soaked separately for $2 \mathrm{hr}$ in $100 \mathrm{ml}$ of each culture filterate of corresponding groundnut seed fungi in four replication of 25 seeds each. 25 treated seeds were placed in sterilized

*Corresponding author: Narendra Kumar, Amity Institute of Biotechnology, Amity University Haryana, Gurgaon Manaser-122413, Haryana, India, Tel: 01242337015; E-mail: narendra.microbiology@rediffmail.com

Received September 01, 2015; Accepted September 16, 2015; Published September 26, 2015

Citation: Kumar N (2015) Preservation of Arachis Hypogea L. Food Seeds by Cuminumcyminum L. Essential Oil. J Plant Pathol Microb 6: 301. doi:10.4172/21577471.1000301

Copyright: $\odot 2015$ Kumar N, et al. This is an open-access article distributed under the terms of the Creative Commons Attribution License, which permits unrestricted use, distribution, and reproduction in any medium, provided the original author and source are credited. 
petridish containing three layers of moist blotters. The number of seeds germinated after 5 days interval for up to 20 days was observed. The controls were maintained by sowing surface sterilized seeds in sterilized blotters.

\section{Isolation of essential oils fromhigher plants and evaluation of their toxicity against test fungi}

The plant parts were surface sterilized by dipping in $70 \%$ ethanol and then washed repeatedly with sterilized double distilled water and hydrodistilled for isolation of volatile constituents separately for 6 $\mathrm{hr}$ in Clevenger's apparatus. After hydro distillation, immiscible oil was separated and dehydrated over anhydrous sodium sulphate. The toxicity of oil and fungicide copper oxychloride and carbendazimwas assessed by using the inverted petri plate technique of Bocher [13] and fungi toxicity measured following Dixit et al [14].

\section{Physico-chemical properties of Cuminumcyminum seed oil}

The oil was characterized by determination of its various physic-chemical properties viz., specificgravity, specificrotation, refractiveindex, acidvalue, saponification number, esternumber, phenolic content and solubility following Langenau [15].

\section{Fungitoxic properties of Cuminumcyminum seed oil}

The MIC of most effective oil was determined by poisoned food technique of Grover and Moore [16]. For studying nature, the oil treated discs of the fungi showing complete inhibition of their mycelia growth upto $7 \mathrm{~d}$ were washed with sterile water and placed again on fresh solidified medium to observe the revival of mycelia growth. The fungi toxic spectrum of the oil was studied against various fungi isolated from groundnut seed samples. In addition, effect of temperature, autoclaving and storage on the fungi toxicity of oil was determined following Pandey et al [17].

\section{Seed dressing}

For seed dressing, a stock solution of Cumin oil was prepared by dissolving $50 \mu \mathrm{l}$ of oil in $1 \mathrm{ml}$ acetone, $200 \mathrm{~g}$ seed was filled in plastic containers and treated with $1 \mathrm{ml}$ stock solution of the oil, dressed by continuous shaking for $5 \mathrm{~min}$ for proper coating. Likewise two preselected contact fungicides, copperoxychloride and carbendazim ( $500 \mathrm{mg} / 100 \mathrm{~g}$ seeds) were also run parallel for comparison purposes. For control set, the seeds were dressed in requsite amount of acetone in place of oil and fungicides. The containers were made airtight and kept at room temperature at $75 \pm 5 \%$ humidity. Obervations for associated mycoflora were made after 6 months.

\section{Fumigant bioassay}

Fresh dried Arachis hypogea seeds kept for food purpose was locally collected in presterilized polyethylene bags. Aliquots of 0.50 $\mathrm{ml}(1000 \mathrm{ppm})$ and $0.76 \mathrm{ml}(1500 \mathrm{ppm})$ of oil and ethylene dibromide were used separately with $400 \mathrm{~g}$ of freshly dried Arachis hypogea seeds in presterilized gunny bags of $500 \mathrm{ml}$ capacity. Likewise, samples of Arachis hypogea to be treated with oil or ethylene dibromide were stored separately in metal containers (tins) of $500 \mathrm{ml}$ capacity. Sterile cotton swabs $(0.50 \mathrm{~g})$ soaked with synthetic fumigants and oil and wrapped in sterilized muslin cloth $(0.75 \mathrm{~g})$ were placed at the bottom of each container of Arachis hypogea seed. Similarly, $400 \mathrm{~g}$ samples of groundnut were treated with phosphine from a $0.50(1000 \mathrm{ppm})$ or 0.76 $\mathrm{g}(1500 \mathrm{ppm})$ of tablet (160 and $240 \mathrm{mg}$ equivalent phosphine) in 500 $\mathrm{ml}$ containers and were stored in a cabinet in the Laboratory at room temperature for 6 months. Each set contained 5 replicates. Mycobiota associated with Arachis hypogea were then isolated by the agar plate technique and the standard blotter technique.

After 6 months storage, phytotoxicity of oil in terms of germination tests were carried out. One hundred seeds were selected randomly from each test lot and aseptically placed in presterilized petridishes containing three layers of moistened blotting paper. All sets were incubated at $28 \pm 2{ }^{\circ} \mathrm{C}$ in a dark chamber and germination was assessed from $2^{\text {nd }}$ to the $9^{\text {th }}$ day. The germinated seeds were allowed to grow for 9 days and radicle and plumule lengths were recorded on the $5^{\text {th }}, 7^{\text {th }}$ and $9^{\text {th }}$ day. One hundred seed from each treatment and control sets were sown in $15 \times 20 \mathrm{~cm}$ earthen pots ( 5 seeds in each pot) containing garden soil. The pots were irrigated at intervals of 4 days. After 45 days, the plants were observed for general health and morphology.

\section{Gas chromatography}

Requisite amount $(0.1 \mu \mathrm{L})$ of pure seed oil of Cuminumcyminum was subjected to GC and GC/MS analysis. The GC was composed of an Agilent Technology $6890 \mathrm{~N}_{2}$ gas chromatograph data handling system equipped with a split-splitless injector (split ratio 50:1) and fitted with a FID using $\mathrm{N}_{2}$ as the carrier gas at flow rate $1 \mathrm{~mL} / \mathrm{min}$. The column was HP- 5 capillary column $(30 \mathrm{~m} \times 0.32 \mathrm{~mm}, 0.25 \mu \mathrm{m}$ film thickness $)$ and temperature program was used as follows: initial temperature of $60^{\circ} \mathrm{C}$ (hold: $2 \mathrm{~min}$ ) programmed at a rate of $3^{\circ} \mathrm{C} / \mathrm{min}$ to a final temperature of $220^{\circ} \mathrm{C}$ (hold: $5 \mathrm{~min}$ ). Temperatures of the injector and FID were maintained at $210^{\circ} \mathrm{C}$ and $250^{\circ} \mathrm{C}$, respectively.

\section{Gas chromatography-mass spectrometry}

The GC-MS analysis of seed oil of Cuminumcyminum was carried out using Perkin Elmer Clarus 500 gas chromatograph (Shelton, CT06484, USA) equipped with a split-splitless injector (split ratio 50:1) data handling system. The column was an RtxR- 5 capillary column $(60$ $\mathrm{m} \times 0.32 \mathrm{~mm}, 0.25 \mu \mathrm{m}$ film thickness). Helium (He) was the carrier gas at a flow rate $1.0 \mathrm{~mL} / \mathrm{min}$. The GC was interfaced with (Perkin ElmerClarus 500) mass detector operating in the EI+ mode. The mass spectra were generally recorded over $40-500 \mathrm{amp}$ that revealed the total ion current (TIC) chromatograms. Temperature program was usedas the same as described above for GC analysis. The temperatures of the injector, transfer line and ionsource were maintained at $210^{\circ} \mathrm{C}, 210^{\circ} \mathrm{C}$ and $200^{\circ} \mathrm{C}$, respectively.

\section{Results}

\section{Storage fungi on food seeds of Arachis hypogea}

Fifteen fungal species were detected from food seeds of Arachis hypogea through blotter method. The most frequent genera were Aspergillus represented by seven species followed by Fusarium (represented by three species). Highest percentage incidences were $F$. moniliforme and A. flavus (7.4 each) followed by Fusarium oxysporum (6.3) F. solani (5.4) and Penicillium glabrum (4.1). Other species of fungi like Alternaria alternata, Aspergillus candidus, A. phoenicus, A. tamarii, A. terreus, A. sydowi, Rhizopus nigricans, Trichothecium roseum, Trichoderma viride occurred less frequently. Seven fungal species of three genera were detected from surface sterilized seeds using moist blotter method. The most dominant genera were Aspergillus (represented by three species). Highest percentage incidence was of $A$. flavus (3.9) followed by A. niger and F. solani (2.5 each). Other forms like Alternaria alternata, Aspergillus sydowi, F. moniliforme and F. oxysporum were infrequent (Table1).

Twelb fungal species belonging to six genera were detected from unsterilized seeds plated over CDA medium. The most dominant 


\begin{tabular}{|l|c|c|c|c|}
\hline \multirow{2}{*}{ Fungi recorded } & \multicolumn{3}{|c|}{ Moist blotter method } & \multicolumn{3}{|c|}{ Czapeksdox agar method } \\
\cline { 2 - 5 } & US & SS & US & SS \\
\hline Alternariaalternate (Fr.) Keissler & 2.4 & 1.2 & 3.2 & - \\
\hline Aspergilluscandidus Pers ex. & 2.1 & - & 3.3 & - \\
\hline A.flavus Link & 8.1 & 3.9 & 19.9 & 6.6 \\
\hline A.nigervan Tieghem & 3.7 & 2.5 & 14.1 & 3.5 \\
\hline A.phoenicis Link & 1.2 & - & - & - \\
\hline A.tamarii Kita & 1.3 & - & 3.2 & - \\
\hline A.terreus Thom & 1.3 & - & - & - \\
\hline A.sydowi (Bainier and Sartory) & 2.4 & 1.0 & 5.0 & 1.0 \\
\hline Thom and Church & 8.1 & 1.2 & 3.0 & - \\
\hline Fusariummoniliforme Sheldon & 6.3 & 1.4 & 6.3 & 3.1 \\
\hline F.oxysporumvon Schlechtendal & 5.4 & 2.5 & 3.2 & 3.6 \\
\hline F.solani (Mart.) Sacc. & 4.1 & - & 11.2 & - \\
\hline $\begin{array}{l}\text { Penicilliumglabrum (Wehmer) } \\
\text { Westling }\end{array}$ & 2.3 & - & - & - \\
\hline Rhizopusnigricans Ehr. & 2.1 & - & 1.3 & - \\
\hline Trichodermaviride Pers.ex.Fr. & 1.2 & - & 3.1 & - \\
\hline $\begin{array}{l}\text { Trichotheciumroseum (Persoon) } \\
\text { Link ex }\end{array}$ & & & & \\
\hline Insect-Trogodermagranarium. & & & & - \\
\hline
\end{tabular}

Insect-Trogodermagranarium.

Table1: Percent incidence of different fungi on the food seeds of Arachis hypogea L.

genera were Aspergillus (represented by five species) followed by Fusarium (three species) and Penicillium glabrum. Highest percentage incidence was of A. flavus (19.9) followed by A. niger (14.1), Penicillium glabrum (11.2) F. oxysporum (6.3) and A. sydowi (5.0). Other fungi like Alternaria alternata, Aspergillus candidus, A. tamarii, F. moniliforme, F. solani, Trichoderma viride, Trichithecium roseum were less common. Five fungal species of two genera were isolated from surface sterilized seeds using CDA medium. The fungi recorded to be internally seed borne were A. flavus, A. niger, A. sydowi, F. oxysporum and F. solani (Table 1). In present investigation, it was observed that in agar plate method fast growing fungi suppressed the development of other fungi making their detection difficult. Slow growing forms like Penicillium, Trichothecium and Trichoderma were better isolated in blotter method as compared to agar method.

\section{Fungaldeterioration of food seed of Arachis hypogea}

The metabolites of most of the test fungi showed inhibitory effects on germination. The rating of fungi based on inhibitory effects on germination put $A$. niger as highly potent. The other fungi in order of potentials for inhibiting seed germination were A. flavus, A. tamari, $F$. moniliforme, A. phoenicus F. solani, F. oxysporum, Alternaria alternata, Aspergillus candidus, Penicillium glabrum, Rhizopus nigricans, Trichothecium roseum. The metabolite of $A$. sydowi and Trichoderma viride showed promotive effect on the germination of seeds of groundnut as compared to control. It is evident from Table 2, that $A$. niger and $A$. flavus caused high degree of mortality and reduction in germination.

\section{Evaluation of essential oils/synthetic fungicide against test organisms}

The essential oil of Cuminum cyminum exhibited absolute toxicity at $500 \mathrm{ppm}$ inhibiting mycelial growth of both test fungi completely, while other oils at these concentrations showed moderate, lower level of fungitoxicity (Table 3).The synthetic fungicide was also found effective in second order after this. The physicochemical properties of the Cuminum cyminum seed oil are recorded in Table 4 . The cumin oil has characteristic pale yellow colour having $0.63 \%(\mathrm{v} / \mathrm{w})$ yield on dry weight basis.

\section{Fungitoxic properties of Cuminum cyminum seed oil}

The MIC of the oil was found to be $400 \mathrm{ppm}$ against both the test fungi. The oil exhibited fungicidal nature at hyper MIC against both the test fungi (Table 5) while it was fungicidal in nature at $500 \mathrm{ppm}$. The Cuminum cyminum seed oil completely inhibited the mycelial growth of 10 fungi at $400 \mathrm{ppm}$ (Table 6) and 14 fungi at $600 \mathrm{ppm}$. The oil it's MIC (400 ppm) was able to inhibit the growth of all 10 discs (each of $5 \mathrm{~mm}$ diam) as well as growth of single mycelia discs of $11 \mathrm{~mm}$ diam, the maximum considered in this study. Thus, fungitoxic potential of oil appeared to be retained heavy inoculums density. The highest temperature $\left(100^{\circ} \mathrm{C}\right)$, autoclaving and storage upto 180 days did not affect the toxicity of the oil against the test fungi and insect (Table 7).

\section{In vivo preservation}

It is evident from Table 8 , table that cumin seed oil completely protected food seeds upto 120 days when seed dressed. The copper oxychloride protected for 60 days and carbandazim protected for 30 days from fungus infestation when seed dressed.

As evident from control sets in Table 9, the groundnut food seeds were associated with 15 fungalspeciesviz. Alternaria alternata, Aspergillus candidus, A. flavus, A. niger, A. phoenicis, A. tamarii, A. terreus, A. sydowi, Fusarium moniliforme, F. oxysporum, F. solani, P.glabrum, Rhizopus nigricans, Trichoderma viride, Trichothecium roseum in both containers.

Food seed stored with oil as preservative had better smell and taste when compared to ones stored with synthetic fungicides and fumigants.

Seeds treated with oil were not associated with fungi in either container. Phosphine was ineffective in control of the fungal species at an $80 \mathrm{mg}$ dose in both containers. At $120 \mathrm{mg}$, it was effective. Ethylenedibromide at 0.25 and $0.38 \mathrm{ml}$ was ineffective.

With respect to germination capacity, the oil treated seeds showed $80-90 \%$, phosphine $70-75 \%$ and ethylene dibromide $55-65 \%$ germination. The seeds of control set ,however exhibited only 45$50 \%$ seed germination (Table 10). The oil had no adverse effect on seed germination, seedling growth and general health of plants when compared with control and synthetic fumigants.

The identified constituents with their respective percentages and Kovat's indices are recorded in Table 11. GC and GC-MS analysis

\begin{tabular}{|l|l|l|}
\hline Fungal species & Percent germination & Percent mortality \\
\hline Alternariaalternata & 65.5 & 34.5 \\
\hline Aspergilluscandidus & 65.6 & 34.4 \\
\hline A.flavus & 24.2 & 75.8 \\
\hline A.niger & 6.0 & 94.0 \\
\hline A.phoenicis & 58.6 & 41.4 \\
\hline A.tamarii & 40.2 & 59.8 \\
\hline A.terreus & 40.6 & 59.4 \\
\hline A.sydowi & 89.4 & 10.6 \\
\hline Fusariummoniliforme & 49.5 & 50.5 \\
\hline F.oxysporum & 35.4 & 64.6 \\
\hline F.solani & 61.4 & 38.6 \\
\hline P.glabrum & 65.9 & 34.1 \\
\hline Rhizopusnigricans & 66.4 & 33.6 \\
\hline Trichodermaviride & 85.3 & 14.7 \\
\hline Trichotheciumroseum & 67.4 & 32.6 \\
\hline Sterilized distilled water (control) & 84.3 & 15.7 \\
\hline
\end{tabular}

Table 2: Effect of culture filterate of fungi on seed germination and seedling mortality of groundnut. 
Citation: Kumar N (2015) Preservation of Arachis Hypogea L. Food Seeds by Cuminumcyminum L. Essential Oil. J Plant Pathol Microb 6: 301. doi:10.4172/2157-7471.1000301

Page 4 of 6

\begin{tabular}{|c|c|c|c|}
\hline \multirow{2}{*}{$\begin{array}{l}\text { Plant species/commercial } \\
\text { fungicides }\end{array}$} & \multicolumn{3}{|c|}{$\begin{array}{l}\text { Percent inhibition of mycelia growth of test fungi } \\
\text { at 500ppm }\end{array}$} \\
\hline & Family & Aspergillusniger & A.flavus \\
\hline AdhatodavasicaNees & Acanthaceae & 95.0 & $100.00^{*}$ \\
\hline Ageratum conyzoides L. & Asteraceae & 76.5 & 64.2 \\
\hline A. houstonianum & Asteraceae & 82.5 & 80.5 \\
\hline Anetumgraveolens $L$. & Umbelliferae & 39.0 & 33.0 \\
\hline Anisomeles ovate R.Br. & Lamiaceae & 64.3 & 60.3 \\
\hline $\begin{array}{l}\text { Artabotryshexpetalous(Lamm) } \\
\text { Merr. }\end{array}$ & Annonaceae & 53.2 & 46.7 \\
\hline Azadirachtaindica A. Juss. & Meliaceae & 43.1 & 38.7 \\
\hline CaesuliaoxillarisRoxb. & Asteraceae & 49.1 & 47.1 \\
\hline Callestemonlanceolatus DC & Myrtaceae & 38.3 & 48.2 \\
\hline Cannabis sativa $\mathrm{L}$. & Cannabinaceae & 12.0 & 9.5 \\
\hline $\begin{array}{l}\text { CinnamomumtamlaNees and } \\
\text { Bbrem }\end{array}$ & Lauraceae & 39.0 & 23.0 \\
\hline Citrus aurantifoliaChristm & Rutaceae & 38.2 & 29.3 \\
\hline Cuminumcyminum(L.) & Apiaceae, & $100.0^{*}$ & $100.0^{*}$ \\
\hline Eucalyptus citriodoraHook & Myrtaceae & 49.1 & 35.8 \\
\hline E.globulus(I.) Herit & Myrtaceae & 60.0 & 34.9 \\
\hline Eupatorium capillifolum(L.) & Asteraceae & 40.0 & 30.9 \\
\hline FeroniaelephantumCorrea & Rutaceae & 49.7 & 60.3 \\
\hline F.limonia(L.) Swingle & Rutaceae & 50.8 & 65.4 \\
\hline Hyptissuaveolens(L.) Poit & Lamiaceae & 47.2 & 27.4 \\
\hline Lantana camera L. & Verbenaceae & 58.3 & 39.1 \\
\hline L.indicaRoxb. & Verbenaceae & 55.7 & 40.0 \\
\hline MenthaarvensisL. & Lamiaceae & 53.9 & 38.6 \\
\hline M.piperataL. & Lamiaceae & 63.3 & 50.3 \\
\hline M.spicataL. & Lamiaceae & 60.3 & 48.2 \\
\hline Murrayakoenighii(L.)Spreng & Rutaceae & 25.8 & 40.1 \\
\hline OcimumadscendensWilld & Lamiaceae & 53.0 & 52.4 \\
\hline O.basilicumL. & Lamiaceae & 40.1 & 50.1 \\
\hline O.canumSims & Lamiaceae & 50.1 & 75.0 \\
\hline O. sanctumL. & Lamiaceae & 49.1 & 52.3 \\
\hline PutranjivaroxburghiiWall & Euphorbiaceae & 90 & 95 \\
\hline TageteserectaL. & Asteraceae & 44.0 & 30.7 \\
\hline ThujaoccidentalisL. & Cuppressaceae & 24.0 & 46.3 \\
\hline Copper oxychloride & *Synthetic fungicide & 94.0 & 90.0 \\
\hline Carbondazim & *Synthetic fungicide & 84.0 & 96.1 \\
\hline
\end{tabular}

Table 3: Evaluation of essential oils of higher plants/fungicides against Aspergillusniger and $A$. flavus.

\begin{tabular}{|c|c|}
\hline Parameters & Values \\
\hline Specific gravity & 0.922 \\
\hline Specific rotation & +10 \\
\hline Refractive index & 1.405 \\
\hline Acid value & 3.45 \\
\hline Saponification number & 153.49 \\
\hline Ester number & 150.04 \\
\hline Phenolic content & Nil \\
\hline Solubility & $\begin{array}{c}\text { Completely miscible with petroleum } \\
\text { etheracetone and } 90 \% \text { ethanol } \\
\text { in 1;1ratio but insoluble in water }\end{array}$ \\
\hline
\end{tabular}

Table 4: Physicochemical properties of Cuminumcyminum seed oil.

\begin{tabular}{|c|c|c|}
\hline Dose of oil in ppm & Aspergillusniger & A.flavus \\
\hline 200 & 30 & 40 \\
\hline 300 & 70 & 80 \\
\hline 400 & $100^{\star}$ & $100^{\star}$ \\
\hline 500 & 100 & 100 \\
\hline 600 & 100 & 100 \\
\hline
\end{tabular}

*Fungicidal

Table 5: Minimum inhibitory concentration ofCuminumcyminum seed oil.

\begin{tabular}{|l|c|c|c|c|}
\hline \multirow{2}{*}{ Fungal species } & \multicolumn{4}{|c|}{ Per cent inhibition of mycelial growth of isolated fungi } \\
\cline { 2 - 5 } & $\begin{array}{c}\text { Sublethal } \\
200 \mathrm{ppm}\end{array}$ & $\begin{array}{c}\text { Lethal } \\
400 \mathrm{ppm}\end{array}$ & $\begin{array}{c}\text { Hyperlethal } \\
600 \mathrm{ppm}\end{array}$ & $\begin{array}{c}\text { Hyperlethal } \\
800 \mathrm{ppm}\end{array}$ \\
\hline Alternariaalternata & 45.6 & 80.0 & 100.0 & 100.0 \\
\hline Aspergilluscandidus & 49.6 & 89.0 & 100.0 & 100.0 \\
\hline A.flavus & 50.0 & 100.0 & 100.0 & 100.0 \\
\hline A.niger & 30.0 & 100.0 & 100.0 & 100.0 \\
\hline A.phoenicis & 40.0 & 100.0 & 100.0 & 100.0 \\
\hline A.tamarii & 48.0 & 100.0 & 100.0 & 100.0 \\
\hline A.terreus & 59.0 & 100.0 & 100.0 & 100.0 \\
\hline A.sydowi & 55.6 & 100.0 & 100.0 & 100.0 \\
\hline Fusariummoniliforme & 40.0 & 100.0 & 100.0 & 100.0 \\
\hline F.oxysporum & 42.0 & 79.6 & 100.0 & 100.0 \\
\hline F.solani & 40.0 & 100.0 & 100.0 & 100.0 \\
\hline P.glabrum & 59.0 & 100.0 & 100.0 & 100.0 \\
\hline Rhizopusnigricans & 54.0 & 100.0 & 100.0 & 100.0 \\
\hline Trichodermaviride & 55.0 & 80.0 & 90.0 & 100.0 \\
\hline Trichotheciumroseum & 65.9 & 95.0 & 100.0 & 100.0 \\
\hline
\end{tabular}

Table 6: Spectrum of Cuminumcyminum seed oil at different doses.

\begin{tabular}{|c|c|}
\hline Physical factors & $\begin{array}{c}\text { Per cent inhibition of mycelial } \\
\text { growth at its MIC }\end{array}$ \\
\hline Temperature $\left({ }^{\circ} \mathrm{C}\right)$ & \\
Time of treatment-60min & 100 \\
$40^{\circ} \mathrm{C}$ & 100 \\
$60^{\circ} \mathrm{C}$ & 100 \\
$80^{\circ} \mathrm{C}$ & 100 \\
$100^{\circ} \mathrm{C}$ & \\
\hline Autoclaving & 100 \\
\hline (15l bs/sq inch pressure at $\left.120^{\circ} \mathrm{C}\right)$ & \\
\hline For 15 min & \\
\hline Storage in days & 100 \\
15 & 100 \\
30 & 100 \\
45 & 100 \\
75 & 100 \\
90 & 100 \\
105 & 100 \\
120 & 100 \\
135 & 100 \\
150 & 100 \\
165 & 100 \\
180 & 100 \\
\hline
\end{tabular}

Table 7: Effect of physical factors on the fungitoxicityof Cuminumcyminum seed oil.

\begin{tabular}{|c|c|c|c|}
\hline \multirow{2}{*}{ Period of incubation in days } & \multicolumn{3}{|c|}{ Appearance of fungal species } \\
\cline { 2 - 4 } & Cumin oil & Copper oxychloride & Carbendazim \\
\hline 30 & - & - & - \\
\hline 60 & - & - & + \\
\hline 90 & - & + & + \\
\hline 120 & - & + & + \\
\hline 150 & + & + & + \\
\hline 180 & + & + & + \\
\hline
\end{tabular}

Table 8: In vivo efficacy of cumin oil and commercial fungicides in preservation of food seeds of Arachis hypogea

of the oil revealed recognition of p-mentha-1, 4-dien-7-al (27.4\%), $\gamma$-terpinene (12.8\%), $\beta$-pinene (11.4\%) and cuminaldehyde (16.1\%) as major compounds.

\section{Discussion}

Several other fungal species were isolated by different workers from groundnut seeds viz., Aspergilluscandidus, A. chevalieri and A. ruber [18]; Mucorsp [19]; Fusarium moniliforme, F. pallidoroseum, 
Citation: Kumar N (2015) Preservation of Arachis Hypogea L. Food Seeds by Cuminumcyminum L. Essential Oil. J Plant Pathol Microb 6: 301. doi:10.4172/2157-7471.1000301

Page 5 of 6

\begin{tabular}{|c|c|c|c|c|c|c|c|c|c|c|c|c|c|c|c|c|c|c|c|c|c|c|c|c|c|c|c|c|}
\hline \multirow{5}{*}{ Fungal species } & \multirow{3}{*}{\multicolumn{4}{|c|}{ control }} & \multicolumn{24}{|c|}{ treatment } \\
\hline & & & & & \multicolumn{8}{|c|}{ Cuminumcyminum oil } & \multicolumn{8}{|c|}{ Phosphine(mg) } & \multicolumn{8}{|c|}{ Ethylene dibromide(ml) } \\
\hline & & & & & \multicolumn{4}{|c|}{0.50} & \multicolumn{4}{|c|}{0.76} & \multicolumn{4}{|c|}{160} & \multicolumn{4}{|c|}{240} & \multicolumn{4}{|c|}{0.50} & \multicolumn{4}{|c|}{0.76} \\
\hline & \multicolumn{2}{|c|}{ A } & \multicolumn{2}{|c|}{$\mathrm{B}$} & \multicolumn{2}{|c|}{ A } & \multicolumn{2}{|c|}{$B$} & \multicolumn{2}{|c|}{$A$} & \multicolumn{2}{|c|}{$\mathrm{B}$} & \multicolumn{2}{|c|}{$A$} & \multicolumn{2}{|c|}{$B$} & \multicolumn{2}{|c|}{$A$} & \multicolumn{2}{|c|}{ B } & \multicolumn{2}{|c|}{ A } & \multicolumn{2}{|c|}{ B } & \multicolumn{2}{|c|}{ A } & & \\
\hline & G & $\mathrm{T}$ & G & $\mathrm{T}$ & G & $\mathrm{T}$ & $\mathrm{G}$ & $\mathrm{T}$ & G & $T$ & G & $\mathrm{T}$ & G & $\mathrm{T}$ & $\mathrm{G}$ & $\mathrm{T}$ & G & $\mathrm{T}$ & G & $\mathrm{T}$ & G & $\mathrm{T}$ & G & $\mathrm{T}$ & G & $\mathrm{T}$ & G & $\mathrm{T}$ \\
\hline Alternariaalternata & + & + & + & + & - & - & - & - & - & - & - & - & + & + & + & + & + & - & + & - & + & + & + & + & + & + & + & + \\
\hline Aspergilluscandidus & + & + & + & + & - & - & - & - & - & - & - & - & + & + & + & + & + & - & + & - & + & + & + & + & + & + & + & + \\
\hline A.flavus & + & + & + & + & - & - & - & - & - & - & - & - & + & + & + & + & + & - & + & + & + & + & + & + & + & + & + & + \\
\hline A.niger & + & + & + & + & - & - & - & - & - & - & - & - & + & + & + & + & + & - & + & - & + & + & + & + & + & + & + & + \\
\hline A.phoenicis & + & + & + & + & - & - & - & - & - & - & - & - & + & + & + & + & + & - & + & - & + & + & + & + & + & + & + & + \\
\hline A.tamarii & + & + & + & + & - & - & - & - & - & - & - & - & + & + & + & + & + & + & + & - & + & + & + & + & + & + & + & + \\
\hline A.terreus & + & + & + & + & - & - & - & - & - & - & - & - & + & + & + & + & + & - & + & - & + & + & + & + & + & + & + & + \\
\hline A.sydowi & + & + & + & + & - & - & - & - & - & - & - & - & + & + & + & + & + & - & + & - & + & + & + & + & + & + & + & + \\
\hline Fusariummoniliforme & + & + & + & + & - & - & - & - & - & - & - & - & + & + & + & + & + & - & + & - & + & + & + & + & + & + & + & + \\
\hline F.oxysporum & + & + & + & + & - & - & - & - & - & - & - & - & + & + & + & + & + & - & + & - & + & + & + & + & + & + & + & + \\
\hline F.solani & + & + & + & + & - & - & - & - & - & - & - & - & + & + & + & + & + & - & + & - & + & + & + & + & + & + & + & + \\
\hline P.glabrum & + & + & + & + & - & - & - & - & - & - & - & - & + & + & + & + & + & - & + & - & + & + & + & + & + & + & + & + \\
\hline Rhizopusnigricans & + & + & + & + & - & - & - & - & - & - & - & - & + & + & + & + & + & - & + & - & + & + & + & + & + & + & + & + \\
\hline Trichodermaviride & + & + & + & + & - & - & - & - & - & - & - & - & + & + & + & + & + & - & + & - & + & + & + & + & + & + & + & + \\
\hline Trichotheciumroseum & + & + & + & + & - & - & - & - & - & - & - & - & + & + & + & + & + & - & + & - & + & + & + & + & + & + & + & + \\
\hline
\end{tabular}

Storage system; G-gunny bags; T-tin containers

Detection method; A-agar plate technique; B-blotter tehnique

+ ; presence of fungi; -absence of fungi

Table 9: Food seed mycoflora of $400 \mathrm{~g}$ seed of Arachis hypogea L. treated with Cuminumcyminum seed oil, Phosphine and ethylene dibromide after 6 months of storage in $500 \mathrm{ml}$ containers.

\begin{tabular}{|c|c|c|c|c|c|c|c|c|c|c|c|c|c|c|}
\hline \multirow{4}{*}{$\begin{array}{l}\text { Period } \\
\text { (days) }\end{array}$} & \multicolumn{14}{|c|}{ Germination $\%$} \\
\hline & \multirow{2}{*}{\multicolumn{2}{|c|}{ control }} & \multicolumn{4}{|c|}{ Adhatoda oil } & \multicolumn{4}{|c|}{ Phosphine (mg) } & \multicolumn{4}{|c|}{ Ethylene dibromide (ml) } \\
\hline & & & \multicolumn{2}{|c|}{0.50} & \multicolumn{2}{|c|}{0.76} & \multicolumn{2}{|c|}{160} & \multicolumn{2}{|c|}{240} & \multicolumn{2}{|c|}{0.50} & \multicolumn{2}{|c|}{0.76} \\
\hline & G & $\mathrm{T}$ & G & $\mathrm{T}$ & G & $\mathrm{T}$ & G & $\mathrm{T}$ & G & $T$ & G & $\mathrm{T}$ & G & $\mathrm{T}$ \\
\hline 2 & 15 & 15 & 15 & 15 & 15 & 15 & 15 & 15 & 15 & 15 & 15 & 15 & 20 & 15 \\
\hline 3 & 25 & 25 & 50 & 50 & 50 & 50 & 40 & 50 & 40 & 35 & 30 & 35 & 35 & 30 \\
\hline 4 & 45 & 45 & 75 & 85 & 75 & 80 & 65 & 70 & 65 & 65 & 60 & 60 & 50 & 35 \\
\hline 5 & 45 & 50 & 80 & 90 & 80 & 85 & 70 & 75 & 70 & 70 & 65 & 65 & 55 & 60 \\
\hline 7 & 45 & 50 & 80 & 90 & 80 & 85 & 70 & 75 & 70 & 70 & 65 & 65 & 55 & 60 \\
\hline
\end{tabular}

G;Gunny bags

$\mathrm{T} ;$ Tin containers

Table 10: Seed germination of Arachis hypogea L. (groundnut) treated with Cuminumcyminum oil, phosphine and ethylene dibromide after 6 months storage of $400 \mathrm{~g}$ samples in $500 \mathrm{ml}$ containers.

\begin{tabular}{|l|l|l|}
\hline Components & Kovat's indices & $\%$ Content \\
\hline p-mentha-1,4-dien-7-al (27.4\%), & 1280 & $27.4 \%$ \\
\hline Y-terpinene & 1068 & $12.8 \%$ \\
\hline$\beta$-pinene & 977 & $11.4 \%$ \\
\hline cuminaldehyde & 1239 & $16.1 \%$ \\
\hline
\end{tabular}

Table 11: Chemical composition of Cuminumcyminum seed essential oil.

F. solani, Microsporum phaseolina and Verticilliumal boatrum; Macrophominaphaseolina, Rhizoctonia solani, Fusarium solani, F. oxysporum, Aspergillus flavus and A. niger [3] but in present investigation 15 fungal species viz.Alternaria alternata, Aspergillus candidus, A. flavus, A. niger, A. phoenicis, A. tamarii, A. terreus, A. sydowi, Fusarium moniliforme, F. oxysporum, F. solani, P. glabrum, Rhizopus nigricans, Trichoderma viride, Trichothecium roseum were isolated. The variation in fungal species may be due to different climatic conditions, isolation periods and different storage containers.

Shaziz et al [3] isolated higher number of fungi by blotter method was used as compared to agar plate and deep-freezing method. Surface sterilization of seeds reduced the incidence of A. flavus and A. niger.

Similarily in present investigation higher number of species were isolated in blotter method and surface sterilization reduced the number of species.

In present investigation, the MIC of Cuminum cyminum seed oil was found to be $400 \mathrm{ppm}$ against both Aspergillus niger and A. flavus. There is a marked variation in the MIC of different plant oils against Aspergillus niger-thus Ocimumadscendens Willd $200 \mathrm{ppm}$ [20], Cymbopogon flexuosus (Steud.) Wats 400 ppm [21], Syzygium aromaticum (L.) Merrill and Perry 200 ppm [22], Cedrusdeodara (Roxb.ex Lambert) G. Don 1000 ppm and Trachyspermumammi (L.) Sprague 500ppm [23]; Putranjivarox burghii Wall 400 ppm [24]. The variation in the MIC of different plant oils may be due to the presence of different chemical constituents.

Wellman [25] mentioned that a fungicide must retain its fungitoxicity at the extreme of temperatures. The fungitoxicity of leaf oil of Adhatoda vasica was found to be thermostable upto $100 \mathrm{C}$ like Ageratum conyzoides [26]; Nardosta chysjatamansi [27]; Putranjivarox burghii ppm [24]. The cumin seed oil retained its fungitoxicity on autoclaving (15 lbs/square inch pressure).This quality of oil will facilitate the isolation of their constituents in active state. 
Citation: Kumar N (2015) Preservation of Arachis Hypogea L. Food Seeds by Cuminumcyminum L. Essential Oil. J Plant Pathol Microb 6: 301. doi:10.4172/2157-7471.1000301

Wellman [25] highlighted that a fungicide should be able to retain its activity during long period of its storage. The fungitoxic factor in the oil of Adenocalyma allicea was lost within $21 \mathrm{~d}$ of storage [28] while persisted for long period in the oil of Ageratum conyzoides [26]; Trachyspermumammi [23] and Putranjivarox burghii ppm [24]. The fungal toxicity was not affected by storage upto 180 days during present investigation. Therefore, this shows that the Cuminum cyminum seed oil can be safely stored at any ambient temperature for long periods without loss in toxicity.

Many reports revealed that, plant metabolites and plant based pesticides appear to be one of the better alternates as they are known to have minimal environmental impact and danger to consumer in contrast to synthetic fungicides $[29,30]$.

Cumin oil was more effective than commercial pesticides during in vivo both during seed dressing and fumigation studies. Seed fumigation method was more effective than seed dressing method, protected seeds of Arachis hypogea kept for food purpose up to 180 days from fungal infestationincreased its shelf life.

\section{Conclusion}

The study revealed that Cumin oil was more fungi toxicants than tested fungicides, thereby indicating the possibility of its exploitation as an antifungal agent for protection of food seeds of groundnut during storage. This may be a fumigant for future as alternate of synthetic pesticides.

\section{Acknowledgement}

Author is thankful to Director Prof. S.M Paul Khurana, Amity Institute of Biotechnology, Amity University Haryana for providing Library and Laboratory facilities.

\section{References}

1. Sharma KK, Bhatnagar-Mathur P (2006) Peanut (Arachis hypogaea L) Methods Mol Biol 343: 347-358.

2. Ameer M, Begum J, Venudevan B, Jayanthi M (2013) Storage Fungi in Groundnut and the Associate Seed Quality Deterioration-A Review. Plant Pathology Journal 12: 127-134.

3. Rasheed S, Dawar S, Ghaffar A, Shahid Shaukat S (2004) Seed borne mycoflora of groundnut. Pak J Bot 36: 199-202.

4. Coats JR (1994) Risks from natural versus synthetic insecticides. Annu Rev Entomol 39: 489-515.

5. Muskett AF (1948) Technique for the examination of seeds for the presence of seed borne fungi. Trans Br Mycol 30: 74-83.

6. De Tempe J (1953) The blotter method of seed health testing. Proc, Int.Seed test Assocn 28: 133-151.

7. Raper KB, Thom C (1949) A Manual of the Penicillia. Boulliere, Tindall and Cox. London pp: 875

8. Gillman JC (1967) A manual of soil fungi. Oxford and JBH publishing co. Calcutta. India.

9. Raper KB, Fennell DI (1965) The genus Aspergillus. The Williams and Wilkins Company,Baltimore. P. 686

10. Booth C (1971) The genus Fusarium. Commonwealth Mycological Institute Kew, Surrey,England, p. 237.

11. Ellis MB (1971) Dematiaceous hyphomycetes. Commonwealth Mycological Institute, Kew, Surrey, England pp: 608.

12. Ellis MB (1976) More dematiaceous hyphomycetes. Commonwealth Mycological Institute, Kew. Surrey, England.

13. Bocher OE (1938) Antibiotics. In: Modern methods of plant analysis. Eds. Peach K and Tracey M.V (ed.). Modern methods of plant analysis vol iii, 651 Springer-Verlag, Berlin
14. Dixit SN, Tripathi NN, Tripathi SC (1978) Fungitoxicity of some seed extracts. Nat Acad Sci Letters 1: 287-288.

15. Langenau EE (1948) The examination and analysis of essential oils, synthetics and isolates. In: Guenther, E (Ed.) The essential oils Vol, 1. Krieger Publishing Co., Hutington, New York pp: 227-348.

16. Grover RK, Moore JD (1962) Toximetric studies of fungicides against brown rot organism, Sclerotiniafructicola and S. laxa. Phytopath 52: 876-880.

17. Pandey DK, Chandra H, Tripathi NN (1982) Volatile fungitoxicity of some higher plants with special reference to that of Callistemon lanceolatus DC. Phytopath Z 105: 175-182.

18. Mukherjee PS, Nandi SK, Nandi B (1992) Deteriorative changes in groundnut seeds in storage. J Mycopathol Res 30: 113-119.

19. Swamy SN, Shambulingappa KG (1994) Provenance effect on seed quality of groundnut in Karnataka. J Oil Seeds Res 11: 204-209.

20. Asthana A, Singh AK (1981) Fungitoxic properties of essential oil of Ocimum adscendens. Journal of Indian Botanical Society Supplement 60: 13.

21. Dixit $V$ (1991) Evaluation of volatile inhibitors from higher plants against storage fungi of Allium cepa. PhD thesis Gorakhpur University, Gorakhpur, India.

22. Khan SA (1993) Control of fungal and insect deterioration of blackgram during storage by some higher plants. PhD thesis Gorakhpur University, Gorakhpur, India

23. Singh J, Tripathi NN (1999) Inhibition of storage fungi of black gram (Vigna mungo $\mathrm{L}$ ) by some essential oils. Flavour Fragrance $\mathrm{J}$ 14: 1-4.

24. Tripathi NN, Kumar N (2007) Putranjiva roxburghii oil-A potential herbal preservative for peanuts during storage. Journal of stored Products Research 43: 435-442.

25. Wellman RH (1967) Commercial development of fungicides. In: Plant pathology Problem and Progress Eds Holtanet al. Indian University Press, Allahabad, India.

26. Dixit SN, Chandra H, Tiwari R, Dixit V (1995) Development of botanical fungicide against blue mould of mandarins. J Stored Prod Res 31: 165-172.

27. Mishra D, Chaturvedi RV, Tripathi SC (1995) The fungitoxic effect of the essential oil of the herb Nardostachys jatamansi DC. Tropical Agri 72: 48-52.

28. Chaturvedi R (1979) Evaluation of higher plants for their fungitoxicity against Helmintho sporiumoryzae. Ph.D Thesis Gorakhpur University, Gorakhpur, India.

29. Ray DP (2008) Activity of essential oils of Ocimum sanctum against Rhizoctonia solani and Fusarium oxysporum. Ann PI ProtecSci16: 537-538.

30. Mangala AC, Kumar A, Aggarwal A (2010) Fungitoxic effect of bio-control agent and botanicals on seed mycoflora and seed germination of oil seed crops. Ann PI Protec Sci 18: 434-437. 